\title{
Performance Analysis of Non-Homogeneous Hybrid Production Lines
}

\author{
Jun Liu, Junping Kong, and Qinying Fan
}

\begin{abstract}
For analyzing performance of non-homogeneous hybrid lines, a new technique of dealing with nonhomogeneous lines is proposed to transform a non-homogeneous production line into a homogeneous line, which is based on decomposition method. The technique extends the applicability of decomposition method to nonhomogeneous lines. The technique is discussed by numerical experiments comparing with aggregation method, what's more, the advantages and disadvantages are specified. The technique complements and develops the system analysis techniques of hybrid production lines.
\end{abstract}

Index Terms-Hybrid system, system performance, Non-homogeneousness, decomposition method.

\section{INTRODUCTION}

It is generally known that a production system is a hybrid system [1]. An amount of work has been devoted to the modeling and analysis of transfer and production lines using analytical methods since the early 1950's because of their economic importance and academic interest. A comprehensive survey presented by Dallery and Gershwin [2] provides extensive and elaborate reviews up to 1992. Li J. et al. [3] offers a supplementary review up to 2007. Readers can also refer to some books [4] on how to model and analyze transfer lines. The two-machine lines are the basis of researching longer production lines. For the models of two machines, such as discrete model, synchronous model, asynchronous model, continuous model, homogeneous model, non-homogeneous model, etc, the approximate solutions have been obtained by some scholars [5]. Now further works have been devoted to the analysis of longer production lines [6]. However, it is very difficult (is not hopeless) to obtain exact analytical solutions of production lines with more than three machines. The major reason is that the system states increase exponentially with the increase of machines. The curse of dimensionality makes such problems intractable even if more powerful computers are available. It appears that "it is difficult to program, ill-behaved, and not extendable to larger problems" [7]. So far to value and analyze the longer production lines, three main approximate techniques have been proposed: decomposition method [7], aggregation method [8] and simulation method. The simulation method is comparatively accurate, however, it is

Manuscript received March 20,2014; revised May 23, 2014. This work was supported by National Natural Science Fund (No.51265032) and Program for Changjiang Scholars and Innovative Research Team in University (No.IRT1140).

The authors are with Lanzhou University of Technology, 730050, Lanzhou, China (e-mail: lzhjliu@126.com, kongjunping425@163.com, 958174577@qq.com). time-consuming. The aggregation method can be utilized directly to analyze homogeneous production line as well as non-homogeneous production line without any transformation. Nevertheless, compared with decomposition method, the aggregation method sometimes has a larger analysis error on some models of production line. A production line for which the processing times or production rates at all machines are equal will be called a homogeneous line.

The idea of decomposition method is to decompose the original production line into a set of two-machine lines, and the behavior of the two-machine lines closely approximates that of the original production line. Many scholars do a great deal of work to increase the efficiency of the technique, such as Dallery Y. and Bihan H. L. [9], Colledani M. and Tolio T. [10], etc. The technique seem to find a balance between complexity and reliability, for example, more simplified and high convergent algorithms such as ADDX,BDDX, etc, are offered and developed [11]-[12]. Moreover, the method has been developed and widely utilized to study production lines with complex construction, e.g. parallel lines [13], assembly/disassembly lines [14], closed loop system [15], etc. In order to use this decomposition technique for analyzing non-homogeneous lines, Gershwin [16] has proposed a transformation of a non-homogeneous line into a homogeneous line. The transformation is as follows. Each machine but one (the fastest one) is replaced by a set of two machines separated by a buffer of capacity 0 . Downstream machine captures the unreliability behavior of the original machine, while the upstream machine represents the processing time. The resulting line is homogeneous and can be analyzed using the decomposition method. Some numerical experiments have been done for testing the transformation technique by Gershwin [6].

The purpose of the paper is to present a new transformation technique. The new transformation technique is tested by numerical experiments based on the decomposition method. The advantages and disadvantages, including the applied circumstance of the transformation are presented by comparing it with aggregation method of Meerkov [17].

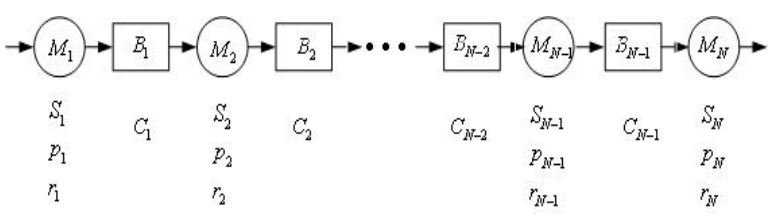

Fig. 1. Production line

\section{PRoblem StATEMENTS}

Fig. 1 is a non-homogeneous production line which 
consists of $N$ machines $M_{i}, i \in\{1,2,3, \cdots, N\}$ and $N-1$ buffers $B_{i}, i \in\{1,2,3, \cdots, N-1\}$. Parts flow from outside of the system to the first machine $M_{1}$, then to the buffer $B_{i}, i \in\{1,2,3, \ldots, N-1\}$, machine $M_{i}, i \in\{2,3, \cdots, N\}$, finally out of the system. The machines are all unreliable. Let $S_{i}, i \in\{1,2,3, \cdots, N\}$ denote the production rate of the machine $M_{i}, i \in\{1,2,3, \cdots, N\}$ and they are different from one another. Then $T_{i}=1 / S_{i}, i \in\{1,2,3, \cdots, N\}$ represent the processing time of each machine. Assume that the up and down times of the machine $M_{i}, i \in\{1,2,3, \cdots, N\}$ are independent and exponentially distributed with means $1 / p_{i}$ and $1 / r_{i} i \in\{1,2, \cdots, N\}$ respectively. $C_{i}, i \in\{1,2, \cdots, N-1\}$ is the buffer size of buffer $B_{i}, i \in\{1,2, \cdots, N-1\}$. Let $\alpha_{i}(t) \in\{0,1\}, i \in\{1,2, \cdots, N\}$ denote the state of the machine $M_{i}, i \in\{1,2,3, \cdots, N\}$ and be a continuous-time Markov process respectively. $\quad \alpha_{i}(t)=1, i \in\{1,2, \cdots, N\} \quad$ indicates the machine $M_{i}, i \in\{1,2,3, \cdots, N\} \quad$ is operational and $\alpha_{i}(t)=0, i \in\{1,2, \cdots, N\}$ indicates the machine is down. Then the system state of the line can be represented by $\pi=\left[n_{1}, \cdots, n_{N-1}, \alpha_{1}, \cdots, \alpha_{N}\right]$ where $n_{i}, i=1,2, \cdots, N-1$ denote the buffer level of $B_{i}, i \in\{1,2, \cdots, N-1\}$. For $M_{i}, i \in\{1,2,3, \cdots, N\}$, $\operatorname{prob}\left[\alpha_{i}(t+1)=0 \mid n_{i-1}(t)>0, \alpha_{i}(t)=1, n_{i}(t)<C_{i}\right]=p_{i} \quad$ and $\operatorname{prob}\left[\alpha_{i}(t+1)=1 \mid \alpha_{i}(t)=0\right]=r_{i}$ exist at time $t$. Assume that there are always parts available at the input of the system and spaces available at the output of the system. Meanwhile, assume that the failures are all dependent.

"Blockage" occurs if the machine $M_{i}, i \in\{1,2,3, \cdots, N-1\}$ is operational and the buffer level $n_{i}, i \in\{1,2, \cdots, N\}$ of the next downstream buffer $B_{i}, i \in\{1,2, \cdots, N-1\}$ reaches its capacity $C_{i}, i \in\{1,2, \cdots, N-1\}$. "Starvation" occurs if the machine $M_{i}, i \in\{2,3, \cdots, N\}$ is operational and the buffer level $n_{i}, i \in\{1,2, \cdots, N\}$ of the adjacent upstream buffer $B_{i}, i \in\{1,2, \cdots, N-1\}$ is zero. When there are not blockage and "starvation", $n_{i}[t+1]=n_{i}[t]+\alpha_{i}[t+1]-\alpha_{i+1}[t+1], i \in\{1,2, \cdots, N\}$. The average production rate of $M_{i}, i \in\{2,3, \cdots, N\}$ is $E_{i}=S_{i} \operatorname{prob}\left[\alpha_{i}=1, n_{i-1}>0, n_{i}<C_{i}\right]$.

Due to the line is a conserve system, the system throughput $E$ of the line in a long run is as follows:

$$
E=E_{i}, i \in\{1,2, \cdots, N\}
$$

The system average surplus $Q_{i}$ of the buffer $B_{i}, i \in\{1,2,3, \cdots, N-1\}$ is as follows:

$$
Q_{i}=\sum_{\pi} n_{i} \operatorname{prob}[\pi], i \in\{1,2, \ldots, N-1\}
$$

The system throughput $E$ and average buffer level $Q_{i}$ are the main system performance parameters of production lines.

\section{A NEW TRANSFORMATION METHOD}

\section{A. Traditional Transformation Technique}

For analyzing non-homogeneous lines, it needs to transform non-homogeneous lines into homogeneous lines to utilize the decomposition method. In 1987, Gershwin [16] proposed a transformation technique which was widely used by scholars later. In the method, each machine but one (the fastest one) is replaced by a set of two machines separated by a buffer of capacity 0 , meanwhile, downstream machine captures the unreliability behavior of the original machine, while the upstream machine represents the processing time. The non-homogeneous lines is shown in Fig. 1, let $T=\min \left(T_{1}, T_{2}, \cdots, T_{N}\right)$. For the machine $M_{i}$ which is not the fastest machine in the system, the transformation proposed by Gershwin is as follows.

$$
\left\{\begin{array}{l}
r_{i}^{2}=r_{i} \\
p_{i}^{2}=\frac{T_{i}}{T} p_{i} \\
\frac{r_{i}^{1}}{r_{i}^{1}+p_{i}^{1}}=\frac{T}{T_{i}} \\
r^{1}, p_{i}^{1}>>r^{2}, p_{i}^{2}
\end{array}\right.
$$

where $p_{i}^{1}$ and $r_{i}^{1}$ is respectively the failure rate and repair rate of upstream machine of the two-machine transformation line, $p_{i}^{2}$ and $r_{i}^{2}$ is respectively the failure rate and repair rate of downstream machine of the two-machine transformation line.

The resulting line utilizes the decomposition method to calculate performance parameters. The idea of the decomposition method is to decompose the line with $N$ machines into $N-1$ two-machine lines $L(i), i \in\{1,2, \cdots, N-1\}$ which consist of an upstream machine $M^{u}(i)$, a downstream machine $M^{d}(i)$ and the buffer $B_{i}, i \in\{1,2, \cdots, N-1\}$. Pseudo-machine $M^{u}(i)$ models the line upstream of $B_{i}, i \in\{1,2, \cdots, N-1\}$, and $M^{d}(i)$ models the line downstream from $B_{i}, i \in\{1,2, \cdots, N-1\}$. The parameters of the pseudo-machines are chosen and updated repeatedly by an iterative algorithm such that the behaviors of the two-machine lines are nearly as same as that of the original line on the whole. Then get the performance parameters of the convergent system by this way. See Fig. 2 for an illustration of a three-machine, two-buffer line.

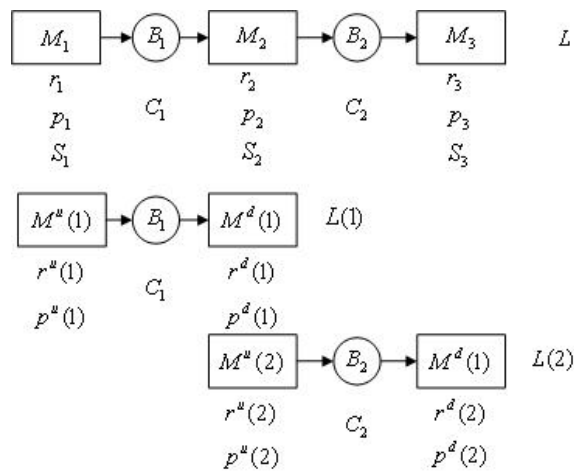

Fig. 2. Illustration of decomposition method. 
In Fig. 2, the line $L$ is decomposed to two pseudo-lines $L(1)$ and $L(2)$. Each machine of the two-machine lines is characterized by its failure and repair rate. The process of the decomposition algorithm is as follows.

Step 1: Initialization: $p^{u}(1)=p_{1}, r^{u}(1)=r_{1}, p^{d}(2)=p_{3}, r^{d}(2)=r_{3}$, $p^{d}(1)=p_{2}, r^{d}(1)=r_{2} \cdot\left(p_{i}\right.$ and $r_{i}$ are the failure rate and repair rate of the machines of the resulting line.)

Step 2: Calculate failure rate $p^{u}(2)$ and repair rate $r^{u}(2)$ of $M^{u}(2)$ so that the behaviors of the two-machine lines are nearly the same as the original line.

Step 3: Then, calculate the parameters of $M^{d}(1)$ by the parameters of $M^{u}(1), M^{d}(2), M^{u}(2)$. Go to step 2 until convergence of the unknown parameters.

\section{B. A New Transformation Techniques}

The paper presents following a new transformation technique based on the transformation proposed by Gershwin.

$$
\left\{\begin{array}{l}
r_{i}^{2}=r_{i} \\
p_{i}^{2}=p_{i} \\
\frac{1}{T} \frac{r_{i}^{1}}{r_{i}^{1}+p_{i}^{1}}=\frac{1}{T_{i}} \\
p_{i}^{1}<<p_{i}^{2}
\end{array}\right.
$$

Let $T=\min \left(T_{1}, T_{2}, \cdots, T_{N}\right) \cdot p_{i}, r_{i}$ are the parameters of original line. In the transformation of (4), downstream machine captures the unreliability behavior of the original machine, while the upstream machine represents the processing time. This is as same as the method proposed by Gershwin. However, the parameters of the downstream machine are the same as the original machine in (4). That is to say, we do not consider the adjustment of failure rate due to enhancing the production rate of original machine which is not the fastest machine. Meanwhile, the very small failure rate of the upstream machine is adopted rather than the very large repair rate in (4). This is because $p_{i}^{1}=\left(\frac{T_{i}}{T}-1\right) r_{i}^{1}$ deduced from (3). Then, if the repair rate $r_{i}^{1}$ is larger, the larger failure rate $p_{i}^{1}$ is obtained. We think the bad influence incurred by enlarging failure rate cannot be eliminated completely by simply enlarging repair rate of machines. So we consider the very small failure rate of machines in (4).

\section{NUMERICAL RESULTS AND DISCUSSION}

For the convenience of comparing the new transformation techniques with the traditional transformation technique proposed by Gershwin [16] and aggregation method of Meerkov [17], the data of the literature [6] are utilized. Note that in order to match the scale of enlarging failure rate in (3), let $p_{i}^{2}=100 p_{i}^{1}$ in (4). The production lines $L 1$ to $L 4$ are examined and their parameters as well as numerical results are presented in Table I to Table IV.
Line $L 1$ consisting of four machines and three buffers exhibits low non-homogeneousness and highly unbalanced buffer capacity; Line L2 consisting of six unreliable machines and five buffers exhibits unbalanced repair rate and failure rate. Line $L 3$ exhibits a stronger non-homogeneousness than line $L 2$, and buffer capacities of L3 are big enough. Line L4 exhibits a high non-homogeneousness.

TABLE I: PRODUCTION LINE $L 1$

\begin{tabular}{cccccc}
\hline \hline & & $M_{1}$ & $M_{2}$ & $M_{3}$ & $M_{4}$ \\
\hline $\boldsymbol{T}_{\boldsymbol{i}}$ & $\mathrm{A}$ & 1.0 & 0.95 & 1.05 & 1.0 \\
$p_{i}$ & $\mathrm{~B}$ & 1.0 & 0.95 & 1.0 & 1.05 \\
$\boldsymbol{r}_{\boldsymbol{i}}$ & & 0.04 & 0.02 & 0.03 & 0.02 \\
$\boldsymbol{C}_{i}$ & 0.08 & 0.04 & 0.06 & 0.04 \\
\hline \hline
\end{tabular}

\begin{tabular}{|c|c|c|c|c|c|}
\hline \multicolumn{6}{|c|}{ NUMERICAL RESULTS } \\
\hline & & $Q_{1}$ & $Q_{2}$ & $Q_{3}$ & $E$ \\
\hline \multirow{4}{*}{ A } & Simulation & 15.3 & 0 & 4.7 & 0.426 \\
\hline & Gershwin's & 18.6 & 0 & 2.0 & 0.487 \\
\hline & $\begin{array}{c}\text { New } \\
\text { technique }\end{array}$ & 14.8461 & 0 & 5.0513 & 0.4217 \\
\hline & Meerkov's & & & & 0.338 \\
\hline \multirow{4}{*}{ B } & Simulation & 14.8 & 0 & 5.9 & 0.434 \\
\hline & Gershwin's & 17.6 & 0 & 3.9 & 0.505 \\
\hline & $\begin{array}{c}\text { New } \\
\text { technique }\end{array}$ & 14.8357 & 0 & 5.5911 & 0.4233 \\
\hline & Meerkov's & & & & 0.342 \\
\hline
\end{tabular}

TABLE II: PRODUCTION LINE L2

\begin{tabular}{|c|c|c|c|c|c|c|}
\hline & $M_{1}$ & $M_{2}$ & $M_{3}$ & $M_{4}$ & $M_{5}$ & $M_{6}$ \\
\hline$T_{i}$ & 0.356 & 0.28 & 0.28 & 0.28 & 0.28 & 0.347 \\
\hline $1 / p_{i}$ & 9.24 & 45 & 45 & 45 & 45 & 9.24 \\
\hline $1 / r_{i}$ & 0.76 & 5 & 5 & 5 & 5 & 0.76 \\
\hline$C_{i}$ & 4 & 2 & 2 & 2 & 4 & \\
\hline \multicolumn{7}{|c|}{ NUMERICAL RESULTS } \\
\hline & $Q_{1}$ & $Q_{2}$ & $Q_{3}$ & $Q_{4}$ & $Q_{5}$ & $E$ \\
\hline Simulation & 1.4 & 0.9 & 0.88 & 0.88 & 2.26 & 2.116 \\
\hline Gershwin's & 1.04 & 0.38 & 0.27 & 0.14 & 0.09 & 2.166 \\
\hline $\begin{array}{c}\text { New } \\
\text { technique }\end{array}$ & 2.437 & 1.144 & 1.023 & 0.904 & 0.786 & 1.919 \\
\hline Meerkov's & & & & & & 2.014 \\
\hline
\end{tabular}

TABLE III: PRODUCTION LINE L3

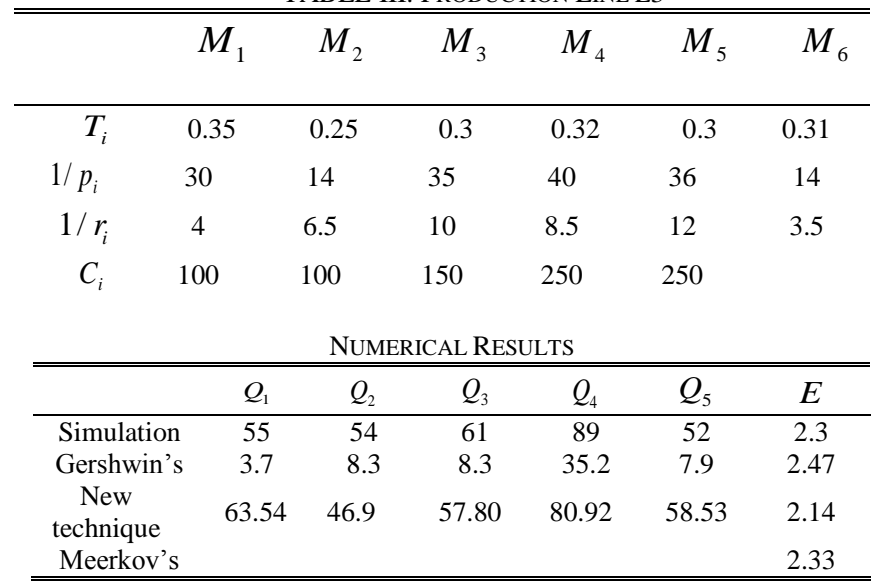


TABLE IV: PRODUCTION LINE $L 4$

\begin{tabular}{|c|c|c|c|c|}
\hline & $M_{1}$ & $M_{2}$ & $M_{3}$ & $M_{4}$ \\
\hline$T_{i}$ & 1.5 & 1 & 0.8 & 1.6 \\
\hline $1 / p_{i}$ & 100 & 140 & 190 & 250 \\
\hline $1 / r_{i}$ & 12 & 18 & 35 & 12.5 \\
\hline$C_{i}$ & 20 & 20 & 15 & \\
\hline \multicolumn{5}{|c|}{ NUMERICAL RESULTS } \\
\hline & $Q_{1}$ & $Q_{2}$ & $Q_{3}$ & E \\
\hline Simulation & 6.1 & 11.5 & 11.8 & 0.556 \\
\hline Gershwin's & 0.2 & 0.1 & 12.9 & 0.595 \\
\hline $\begin{array}{l}\text { New } \\
\text { technique }\end{array}$ & 11.7503 & 9.1432 & 5.7024 & 0.3931 \\
\hline Meerkov's & & & & 0.536 \\
\hline
\end{tabular}

The following conclusion can be drawn from the numerical results of Table I to Table IV. Note that these numerical examples are only a few among those we tested.

For lines with low non-homogeneous characteristics, it can be seen that compared with simulation results respectively in Table I, the better results or results with less error on the throughput $E$ and the system average surplus $Q_{i}$ are obtained by the new method. Line $L 1$ is a typical line for which the new method works well. However, with the increasing non-homogeneousness of lines, e.g., $L 2$ and $L 4$, Gershwin's method has smaller analysis errors on the throughput $E$, whereas has larger analysis errors on the surplus $Q_{i}$ on the whole.

This is because if a very large repair rate $r_{i}^{1}$ is considered, a large failure rate would be obtained due to $p_{i}^{1}=\left(\frac{T_{i}}{T}-1\right) r_{i}^{1}$ (please refer to (3)). Whereas, as mentioned in former section, we think the bad influence incurred by enlarging failure rates cannot be eliminated completely by simply enlarging repair rates of machines from the system viewpoint of lines. That is to say, the enlarged failure rates of machines result in stronger impact on the system performance than the equal-scale enlarged repair rates, especially for the lines with low non-homogeneousness. So for the lines with low non-homogeneousness $L 1$, the numerical results with smaller error are obtained by the new method in which the small failure rate is considered.

However, with the increasing non-homogeneousness of lines, the treatment that the large repair rate is considered is better able to reflect the increasing of blockage and starvation incurred by increasing non-homogeneousness than the treatment that the small failure rate is considered because of $p_{i}^{1}=\left(\frac{T_{i}}{T}-1\right) r_{i}^{1}$. So Gershwin's method has smaller analysis errors on the throughput $E$ for lines with high non-homogeneousness. Nevertheless, the enlarged failure rates of machines have a strong impact on the surplus of buffers. So for lines with high non-homogeneousness, the surplus $Q_{i}$ obtained by Gershwin's method on the whole has larger error than results obtained by the new method due to enlarged failure rates. Note that the decomposition method and Gershwin' method, including the new transformation technique offered in the paper, are all approximation analysis techniques after all, so that the system performances are only estimated approximately. In addition, For line $L 3$, although the line has high non-homogeneousness, the numerical results exhibit the similar features of lines with low non-homogeneousness. This is because the capacities of buffers in the line $L 3$ are very large so that the blockage of the system is few and the system exhibits the similar characteristics of low non-homogeneous system. Furthermore, it can be drawn from the numerical results of Table I to Table IV, Meerkov's method is suitable to analyze the highly unbalanced lines which is with high non-homogeneousness and has smaller analysis error on the throughput of the line.

\section{CONCLUSION}

A new technique has been proposed to analyze the performance of hybrid production lines. The technique was devised to facilitate the analysis of non-homogeneous lines. The comparison analysis between the technique and the Meerkov's aggregation technique was done by numerical experiments. It can be drawn that the new technique has advantages to analyzing the lines with non-homogeneousness or high occurrence probability of blockage and starvation. The new technique develops the system analysis methods of production lines with unreliable machines.

\section{REFERENCES}

[1] G. Labinaz, M. M. Bayoumi, and K. Rudie, "A survey of modeling and control of hybrid systems," Annual Reviews in Control, vol. 21, pp. 79-92, 1997.

[2] Y. Dallery and S. B. Gershwin, "Manufacturing flow line systems: a review of models and analytical results," Queueing System, vol. 12, pp. 3-94, 1992.

[3] J. Li, D. E. Blumenfeld, N. Huang, and J. B. Alden, "Throughput analysis of production systems: recent advances and future topics," International Journal of Production Research, vol. 47, issue 14, pp. 3823-3851, 2009.

[4] T. Altiok, Performance analysis of manufacturing systems, New York: Springer, 1996.

[5] J. Li and S. M. Meerkov, Production System Engineering, New York: Springer, 2008.

[6] Y. Dallery, R. David, and X. L. Xie, "Approximate analysis of transfer lines with unreliable machines and finite buffers," IEEE Transactions on Automatic Control, vol. 34, no. 9, pp. 943-953, 1989.

[7] S. B. Gershwin, "An efficient decomposition method for the approximate evaluation of tandem queues with finite storage space and blocking," Operations Research, vol. 35, no. 2, pp. 291-305, 1987.

[8] B. Ancelin and A. Semery, "Calcul de la productivite d'une ligne integree de fabrication: CALIF, une méthode analytique industrielle," RAIRO APPII, vol. 21, no. 3, pp. 209-238, 1987.

[9] Y. Dallery and H. L. Bihan, "An improved decomposition method for the analysis of production line with unreliable machines and finite buffers," International Journal of Production Research, vol. 37, no. 5, pp. 1093-1117, 1999.

[10] M. Colledani and T. Tolio, "A decomposition method to support the configuration/reconfiguration of production systems," CIRP Annals, Manufacturing Technology, vol. 54, no. 1, pp. 441-444, 2005.

[11] M. H. Burman, "New results in flow line analysis," Thesis (PhD), OR Center, MIT, 1995.

[12] H. B. Le and Y. Dallery, "A robust decomposition method for the analysis of production lines with unreliable machines and finite buffers," Annals of Operations Research, vol. 93, pp. 265-297, 2000.

[13] J. Li, "Modeling and analysis of manufacturing systems with parallel lines," IEEE Transaction on Automatic Control, vol. 49, pp. 1824-1829, 2004.

[14] S. B. Gershwin and M. H. Burman, "A decomposition method for analyzing inhomogeneous assembly/disassembly system," Annals of Operation Research, vol. 93, pp. 91-115, 2000.

[15] J. Li, "Performance analysis of production systems with rework loops," IIE Transaction, vol. 36, pp. 755-765, 2004. 
[16] K. Dhouib, A. Gharbi, and S. Ayed, "Simulation based throughput assessment of non-homogeneous transfer lines," International Journal of Simulation Model, vol.8, no.1,pp.5-15, 2009

[17] J. Li and N. J. Huang, "Modeling and analysis of a multiple product manufacturing system with spit and merge," International Journal of Production Research, vol. 43, pp. 4049-4066, 2005.

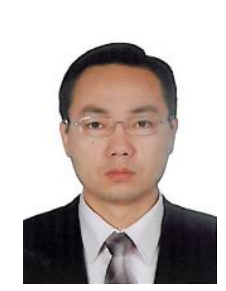

Liu Jun is currently a professor in Lanzhou University of Technology, Lanzhou, China. He received his Ph. D. degree in engineering from Zhejiang University, Hangzhou, China in 2005. His current research interests include complex manufacturing system, production scheduling and control, lean production, etc.

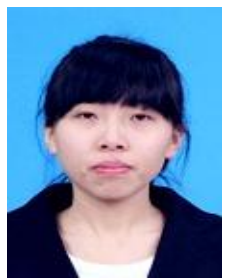

Junping Kong received her bachelor degree from Lanzhou University of Technology, Lanzhou, China, in 2012. She is currently a graduate student. Her current research interests include complex manufacturing system, production scheduling and control, etc.

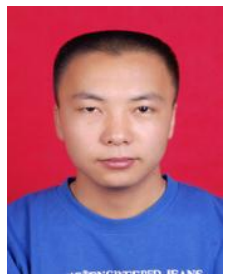

Qinying Fan received his bachelor degree from Lanzhou University of Technology, Lanzhou, China, in 2012. His is currently a graduate student. His current research interests include complex manufacturing system, production scheduling and control, etc. 
